\title{
Metallic stents in the airway: should we continue to use them and can we remove them?
}

\author{
Andrés F. Sosa • Gaëtane C. Michaud
}

Published online: 21 December 2012

(C) Springer Science+Business Media New York 2012

\begin{abstract}
Metallic stents have proven to be valuable tools for the management of both malignant and benign airway obstruction. Their use has become popular worldwide as has our knowledge of their long-term effects on the airway. It is essential to understand the implications of placing a metallic stent since they are not devoid of potentially significant complications. It is also important to acknowledge that metallic stents are not always easily removed and a lack of familiarity with the complexities of their removal can have serious consequences. In general, their use should be reserved for malignant airway obstruction or in rare cases of benign disease where all other options have been ruled out.
\end{abstract}

Keywords Stent $\cdot$ Airway obstruction ·

Airway complications

\section{Introduction}

After the initial conception of endoscopic endobronchial stenting by Brunings and Albrecht in 1915 [1], further developing of this therapeutic intervention was stagnant for several decades. In 1965, Montgomery designed a Ttube made of silicone that has an external limb that anchors the stent in the trachea to prevent it from migrating [2]. In 1990, Dumon truly set into motion a whole new array of

\footnotetext{
A. F. Sosa

Pulmonary, Allergy and Critical Care Medicine, University

of Massachusetts Medical School, 55 Lake Av. North,

Worcester, MA 01655, USA

e-mail: andres.sosa@umassmemorial.org

G. C. Michaud ( $\square)$

Pulmonary and Critical Care Medicine, Yale School of Medicine,

15 York Street, LCI 100C,

New Haven, CT 06510, USA

e-mail: gaetane.michaud@yale.edu
}

modifications with his "dedicated tracheobronchial silicone stent," and between 1987 and 1989 a total of 66 patients were treated with excellent results [3].

The Dumon stent is made of silicone with studs placed on the outer surface to prevent migration. After introduction of the Dumon stent, metallic stents followed quickly. Their design was based on the already well-developed endovascular stent. Their innovation brought the advantage of easy placement with flexible bronchoscopy precluding the need for rigid bronchoscopy. As more physicians started placing metallic stents, the complications also became more evident, including stent fracture, granulation tissue formation with more occlusion of the airway, and infection [4]. Metallic stents may be very difficult to remove, even by the most experienced bronchoscopist, sometimes to the point of needing both endoscopic and surgical extraction [5•]. The risk of severe complications of metallic stents resulted in the Food and Drug Administration (FDA) issuing a public health notification recommending its use in benign disease only after exploring all other treatment options [6].

It has often been touted that the ideal stent should conform to the airway anatomy and yet be able to counteract a sufficient extrinsic pressure. It should be available in different sizes and lengths, and be easy to place and remove when necessary. The ideal stent must be well tolerated without the risk of granulation tissue formation or infection and should not fracture or migrate. In addition, cost must be contained $[4,7,8]$. None of the currently available stents fit all of these criteria. However, this is an evolving field and more innovation is sure to come.

\section{Metallic stents for malignant airway disease}

Approximately $20-30 \%$ of patients with lung cancer will develop complications associated with airway obstruction 
[4]. Patients may present with lobar or complete lung collapse, post-obstructive pneumonia and severe dyspnea.

Malignant airway obstruction is now a major indication for the use of metallic stents. In this patient population, metallic stents have proven to relieve symptoms, and reduce work of breathing as well as health care utilization [9-12]. Although not curative, stents are effective means of palliating central airway obstruction and are often used in conjunction with other therapies to eradicate tissue, including mechanical debridement of exophytic lesions with the rigid bronchoscope, balloon dilatation, electrocautery, argon plasma coagulation (APC), photodynamic therapy (PDT), cryotherapy and brachytherapy [13]. Airway stabilization with a stent may also allow time for palliative chemotherapy and radiation to take effect. In the context of airway intervention with stent placement, survival does not appear to differ from patients without airway obstruction [14].

Airway obstruction may be intrinsic, extrinsic or mixed. Stents offer support from extrinsic compression and malacia, as well as provide a barrier effect against endoluminal tumor growth and fistula formation. Both covered and uncovered metallic stents are commercially available. A thin, usually polyurethane film over the stent provides a barrier to tumor infiltration through the metallic mesh. In the case of intrinsic obstruction, uncovered stents have no role, as there is a high probability of tumor regrowth through the stent. With respect to pure extrinsic compression, uncovered stents may provide better mucocilliary clearance and allow ventilation into airways either completely or partially covered by the stent.

As stated above, a balance must be sought between the beneficial palliative effects of stenting and the potential for complications necessitating removal. The development of granulation tissue can beget further airway compromise and renders removal quite complicated, particularly a month or two after deployment [15•]. When placing a metallic stent, one must be as certain as possible that the stent will not have to be removed. Need for future removal is often very hard to predict, so the use of silicone stents should be advocated when feasible.

\section{Metallic stents for benign airway disease}

Despite the FDA advisory in 2005 [6], metallic stents are frequently used for the treatment of benign central airway obstruction [16]. Their ease of placement under flexible bronchoscopy has made them particularly attractive, especially since only $18 \%$ of fellowship programs offer rigid bronchoscopy during their training, with only $48 \%$ of those achieving competency [17]. Other advantages of metallic stents include a thinner wall that allows for greater airway diameter, better conformation to airway, maintenance of ventilation to airways partially or completely covered by the stent, and preservation of mucocilliary clearance, as they also allow for epithelial growth within the stent [8].
It is now widely accepted that metallic stents should be a last resort for benign airway disease in the majority of cases $[16,18$, 19•, 20]. Silicone stents and/or surgical intervention should always be contemplated first. That notwithstanding, metallic stents have been and can be used under special circumstances for the treatment of benign airway disease [16, 21].

Some of the potential applications for benign airway disease are represented in Table 1. These indications include postintubation and post-tracheostomy tracheal stenosis, postpneumonectomy bronchopleural fistula, post-pneumonectomy syndrome, stenosis after lung transplant, tuberculosis, goiter, arterial aneurysms, tracheobronchomalacia, amyloidosis and inflammatory diseases (sarcoid, ANCA-associated vasculitis with granulomatosis, relapsing polychondritis). Immediate good results are invariably reported, but often at the expense of severe long-term sequelae $[4,7,16,20,21]$.

It is very important to keep in mind that many of these conditions can be treated with a silicone stent and/or surgery, and ease of placement or lack of rigid bronchoscopy training should not be the only indication for placement of a metallic stent.

\section{Metallic stents for tracheoesophageal fistulas}

Malignant tracheoesophageal fistula is a serious, incapacitating complication that carries a poor prognosis. Patients may develop severe dyspnea, dysphagia and recurrent aspiration pneumonias. Esophageal stenting may cause secondary

Table 1 Indications for stent placement*

\begin{tabular}{ll}
\hline Benign Disease & Malignant Disease \\
\hline Extrinsic Compression & Extrinsic compression \\
Postpneumonectomy syndrome & Endoluminal disease \\
goiter & Mixed compression and \\
& tumor infiltration
\end{tabular}

arterial aneurysms

Endoluminal Disease and Stenosis

Stenosis related to artificial airways

bronchopleural fistulas

stenosis after lung transplant

tuberculosis

amyloidosis

papillomatosis

sarcoid

ANCA-associated vasculitis with granulomatosis

Tracheobronchomalacia

congenital

acquired

*This table does not include all indications for stent placement 
airway compromise by extrinsic compression when the stent is fully deployed. Airway stenting with or without esophageal stenting has been performed successfully in cases of malignant tracheoesophageal fistulas; it has been shown to improve symptoms and quality of life [22, 23]. In a study by Herth, airway, and in the majority of cases esophageal, stenting using self-expanding nitinol stents was performed in 112 patients with malignant tracheoesophageal fistulas. Stenting resulted in improvement of quality of life scores, and the overall mean survival in the study population was 236.6 days [22].

\section{Types of metallic stents}

Metallic stents can be uncovered (Wallstent, Ultraflex, Palmaz, Gianturco, Strecker) or covered (Ultraflex, Wallstent). Hybrid stents (Aero, Silmet, Rüsch) are fully covered and try to achieve the maximal luminal patency and conformation of metallic stents with the ease of removal of silicone stents. Metallic stents are introduced in a compressed form and can be balloon-expandable (Palmaz, Strecker) or selfexpandable, in which case the stent gains its original shape by its inherent elasticity [24]. The most commonly used metallic stents today are made of nitinol, a nickel-titanium alloy that has "shape memory effect". Nitinol stents are easily deformable when cold and regain their original shape at body temperatures. Their plasticity allows the tracheal diameter to narrow appropriately with cough, aiding mucocilliary clearance [24-26]. The most common types of metallic stents are represented in Table 2.

\section{Balloon-expandable stents}

The currently available balloon-expandable metallic stents include the Strecker (Boston Scientifc Corp; Watertown, MA) and the Palmaz stent (Johnson \& Johnson Interventional Systems; Warren, NJ). The Palmaz stent is mostly used in children because of its smaller size. Balloonexpandable stents are mounted on a saline-filled balloon,

Table 2 Types of metallic stents

\begin{tabular}{ll} 
Types of metallic stents & \\
\hline Balloon expandable & Uncovered \\
Palmaz & Palmaz \\
Strecker & Strecker \\
Self-expandable & Wallstent \\
Wallstent & Gianturco \\
Gianturco & Ultraflex \\
Ultraflex & Covered \\
Aero & Wallstent \\
& Ultraflex \\
& Aero \\
\hline
\end{tabular}

constructed to expand the stent to its original dimension once placed in the desired position within the airway lumen. These are stents originally designed for intravascular use, where intraluminal fluid exerts additional pressure to maintain stent patency. In the airway, the external pressure is not counteracted by intraluminal pressure and these stents tend to fracture. Furthermore, if embedded in mucosa and granulation tissue develops, they may completely collapse the airway when removed. In 1995, Becker described his experience with 95 patients. The stents placed included 36 Strecker stents, 77 Nitinol stents and 14 other types. The author noted many complications from the Strecker stent, mainly stent fracture and airway occlusion, therefore ceased using it altogether, and even warned against the use of other expandable stents, in particular the Palmaz [24].

\section{Self- expanding stents (SEMS)}

The Gianturco-Z stent (Cook Inc; Bloomington, IN) was one of the first to be commercially available. This selfexpanding stent is made of stainless steel with barbs on opposing sides to prevent migration by anchoring to the endobronchial mucosa. The barbs also make it extremely difficult to remove once fully deployed. It lacks the elasticity of nitinol stents and does not readily conform to the airway. The Gianturco stent has been associated with the highest risk of airway perforation during cough, due to its extreme stiffness under compression, resulting in the highest pressure over a small surface area [26]. Perforations into the esophagus and pulmonary arteries have been documented [24, 27]. Because of its high complication risk, the Gianturco stent has fallen out of favor and its use is discouraged by most authorities [7, 24].

The Wallstent (Boston Scientific Corp) is made of cobaltbased super-alloy monofilament. This type of stent is flexible and compressible and available with a polyurethane covering. Wallstents respond to cough with excessive changes in length that may irritate the airway mucosa and induce formation of granulation tissue [26]. With time, these stents may become embedded in airway mucosa, rendering them extremely difficult to remove. In one study, the late complications observed included stent migration (12\%), inflammatory granulation or tumor regrowth $(36 \%)$, and symptomatic retention of secretions (38\%) [28].

The Ultraflex stents (Boston Scientific Corp; Natick, MA) are fabricated either uncovered or covered by polyurethane. These stents were recently withdrawn from the market in the US. The uncovered stent is now available again. This is another self-expanding stent that can be placed with a flexible bronchoscope using a guide-wire. The Ultraflex stent is made of nitinol, and therefore conforms well to airway anatomy, and thanks to it elasticity, this stent narrows appropriately with cough, aiding in airway clearance. These 
stents can be pulled proximally to reposition after deployment by grasping a string that grabs the stent like a purse. Repositioning stents that have been deployed too proximally proves much more challenging, as the distal purse string must be grasped and the stent collapsed while pulling distally. Miyazawa reported on the insertion of fifty-four Ultraflex stents in 34 patients with inoperable airway stenosis. There was improvement in clinical, endoscopic examination and pulmonary function on days 1, 30 and 60 after stent placement. Tumor ingrowth and tumor overgrowth were the most commonly observed complications ( $21 \%$ and $24 \%$ respectively) [25]. These stents were also available with a polyurethane covering that made them useful for endotracheal disease, preventing tumor growth through the stent. Stent fracture, infection and long term occlusion by granulation tissue are also problems encountered with these stents, and removal may become extremely difficult $[15 \cdot, 29]$.

The Aero (Merit Medical Endotek; South Jordan, UT) stent is a nitinol stent that is fully covered by a polyurethane coating that should minimize the possibility of tissue ingrowth and granuloma formation. Its "dog-bone" shape is meant to reduce migration. Either end of the stent is $2 \mathrm{~mm}$ wider than the main body to achieve better stabilization. The edges of the stent are vaulted inwardly to prevent it from becoming embedded in the airway mucosa. The stent is cut from a single tube of nitinol, preventing further elongation or foreshortening during deployment. It has a hydrophilic coating in the inner lumen to prevent mucous adherence. It may be placed with flexible bronchoscopy using a guidewire, or under direct visualization using a preloaded delivery system $[30,31]$.

In one study involving the AERO stent, fifty-six patients underwent stent placement. Physicians rated ease of deployment at 3.6 on a scale of $1-4$ ( 4 being excellent). Patients were followed for 90 days, during which time the stent proved to be effective for the relief of airway obstruction and dyspnea. There was a high migration rate (28\%), while other stent-related complications occurred at a lower rate, and included minimal granulation tissue formation (5\%) and infection (4\%) [31].

\section{Complications and the FDA advisory}

Over time, the metallic stents may become completely embedded in the airway wall, and obstruction by excessive granulation tissue, fracture, erosion into the mediastinum or adjacent structures may occur [15•, 32-34]. These complications become more evident in benign airway disease, given the longevity of these patients compared to patients with malignancy [35]. These severe complications resulted in the Food and Drug Administration (FDA) advisory of
2005. In the FDA advisory, it was recommended that metallic stents be used with benign airway disease "only after thoroughly exploring all other treatment options (such as tracheal surgical procedures or placement of silicone stents)". The FDA advisory also warned against the placement and removal of metallic stents by physicians not experienced in this procedure [6].

In a single institution retrospective analysis of all patients undergoing stenting for malignant airway obstruction, 175 patients underwent 195 stent procedures. Ultraflex and Wallstents were used in $60 \%$, Aero stents in $16 \%$ and Dumon silicone bronchial and Y-stents were used in $24 \%$. Seventy-three patients $(41 \%)$ developed lower respiratory tract infections that led to significant morbidity and mortality $(23 \%$ died within 14 days of infection). Univariate and multivariate analysis demonstrated that only Aero stents seemed to increase the risk of infection significantly $(p=0.041)$. Twenty-seven (16\%) stent migrations occurred; however, only tubular silicone stents were significantly associated with migration $(p=0.007)$. Granulation tissue formation occurred in $19 \%$, with a median time to granulation of 1.4 months. Infections were associated with the development of granulation $(p<0.001)$. Mucous plugging requiring intervention was reported in $25 \%$ of 195 stent procedures. Structural fractures were seen in three Ultraflex and a single aero stent [36].

In another retrospective review of Ultraflex and Wallstents placed for both benign and malignant airway disease, the observed complications included infection in $15.9 \%$, obstructive granulomas in $14.6 \%$ and migration in $4.7 \%[21]$.

In a retrospective study describing a single institution's experience with metallic stents for benign airway disease, twenty-eight patients had metallic stents removed for complications regarding obstructing granulation tissue, stent migration, wire fracture with granulation, inadequate size and dynamic obstruction. One case involved erosion of a Wallstent through the left bronchial wall. The stent was juxtaposed to the pulmonary artery and the patient required a left pneumonectomy [33].

Other observed serious complications of metallic stents include airway perforation with mediastinal stent migration, aorto and tracheopulmonary artery fistula, and a case of fatal hemorrhage with tracheo-innominate fistulization [27, 32, 37, 38].

It is important to point out, that the most severe complications of airway stents in the vast majority of cases are described in either benign airway disease or patients with a malignancy surviving for an extended period. Even though silicone stents also have significant complications, they do not compare with the potential severity of the complications from the metallic stents. 


\section{Removal}

Removal of metallic stents is generally simple in a newly placed stent. However, we have observed severe granulation tissue formation even after a few days of placement. With time, metallic stents become embedded in the airway mucosa, and stimulate the growth of obstructing granulation tissue that may in turn cause lobar or whole lung collapse. The volume loss can then result in recurrent post-obstructive pneumonias. Metallic stents may perforate the airway or contribute to recurrent infections. It becomes necessary to remove the stent when these complications occur, and removal at this point becomes extremely difficult [15•, 33-36].

Some authors report removal of metallic stents with relative ease using rigid bronchoscopy and an alligator forceps. The stent is grasped and lifted as the rigid bronchoscope is advanced coaxially, separating the stent from the airway wall [39]. Others report finding stents so deeply covered by mucosa that they had to remove them in a piecemeal fashion, sometimes also doing an open tracheostomy for removal of the unwound wire [5•]. At times, pneumonectomy has been the only option to prevent erosion of a stent into the pulmonary artery [33]. On occasion, stents cannot be removed, even by the most experienced bronchoscopists [15•].

We have encountered significant difficulties when removing stents that have been in place for a prolonged period of time. We recently encountered a patient diagnosed with stage IIIB NSCLC who had two Ultraflex stents placed in his Bronchus Intermedius three years prior to presentation. The indication for metallic stents seemed appropriate at the time. However, he developed recurrent pneumonias and near complete occlusion of all lobes by severe granulation (Fig. 1). The stents were both removed in piecemeal fashion in a two-step procedure. On day 1 , we performed cryotherapy of all granulation tissue to loosen

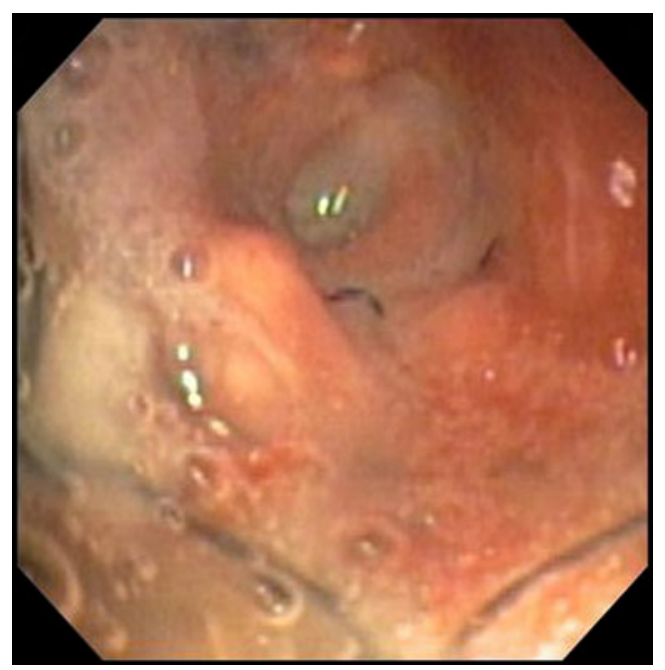

Fig. 1 Ultraflex stents in bronchus intermedius with obstructing granulation tissue the stents, and on day 2, we removed the stents. In another case, a patient had multiple stents placed over a period of 10 years for tracheobronchomalacia and tracheobronchomegaly. The initial stent was a Wallstent, with an Ultraflex placed once granulation tissue developed, and eventually a silicone stent. As the stents were deeply embedded in the mucosa, it took several procedures to remove them, and the procedures were complicated by bleeding and loss of airway integrity. Due to the highly inflammatory nature of the removal process, the patient required intubation for pneumatic stenting in order to provide a "stent holiday" prior to re-establishing patency with a silicone Y-stent.

In a recent retrospective analysis by Alazemi, 55 SEMS were removed from 46 patients. Eighty percent of the patients had benign tracheal disorders. Stents had been in place on average for 292 days. Granulation tissue with highgrade stenosis was the most common indication for removal. Thirty-one stents $(56 \%)$ required additional interventions for removal including ablation of granulation tissue with argon plasma coagulation or electrocautery. Multiple sessions were needed for $25 \%$ of cases. One case required a thoracotomy and bronchotomy that unfortunately was complicated by wound dehiscense of the anastomosis and eventually culminating in pneumonectomy. Health-care resource use was also documented. Only $22 \%$ of the procedures were performed on an outpatient basis, with overall mean duration of hospital stay of 3.5 days. Thirty-nine percent required an ICU admission and median hospital cost per encounter was $\$ 10,700$ (range from $\$ 3,700$ to $\$ 69,800$ ). Patients who had their SEMS placed for benign conditions were more likely to develop complications, hospitalization, longer hospital stay and higher cost with stent removal $[15 \bullet]$.

\section{Conclusion and recommendations}

Should we continue to use metallic stents? The short answer is yes. Metallic stents provide an effective means of palliating airway obstruction. They can be placed safely under flexible bronchoscopy on an outpatient basis. However, even their use in malignant airway obstruction needs careful consideration. Some patients with advanced malignancy can live long enough to develop long-term complications. The use of metallic stents for benign airway disease is discouraged. In the vast majority of benign airway obstructions, metallic stents should be the last resort, only placed after other alternatives have been exhausted (i.e. silicone stents or surgery). In all instances, metallic stents should only be placed by experienced bronchoscopists, familiar with their indications and proper placement, as well as their potential short and long-term complications.

Can we remove metallic stents? Again, the short answer is yes. The removal of metallic stents may be a simple 
procedure performed under flexible bronchoscopy [40, 41]. This may be achievable when they have been in for less than 30 days [15 $]$. After longer periods of time, removal becomes much more difficult, and should be performed by personnel experienced in rigid bronchoscopy and a variety of airway ablative techniques. When removing metallic stents, flexible bronchoscopy may not be an option. One should also have a definite plan on how to proceed. A multiple step procedure may be indicated; the choice of endoscope should be clear. Complications must be anticipated (i.e bleeding, infection, respiratory failure from reocclusion and need for re-stenting). We advocate that these complex procedures be referred to tertiary centers, equipped with multidisciplinary teams experienced in management of complex airway disease.

Disclosure No potential conflicts of interest relevant to this article were reported.

\section{References}

Papers of particular interest, published recently, have been highlighted as:

- Of importance

1. Brunings W, Albrecht W. Direkte endoskopie der luft - und speisewege. Stuttgart: Enke; 1915. p. 134-8.

2. Montgomery WW. T-tube tracheal stent. Arch Otolaryngol. $1965 ; 82: 320-1$.

3. Dumon JF. A dedicated tracheobronchial stent. Chest. 1990;97:328-32.

4. Lee P, Kupeli E, Mehta A. Airway stents. Clin Chest Med. 2010;31:141-50.

5. • Rampe AM, Silvestri GA, Gillespie MB. Combined endoscopic and open approach to the removal of expandable metallic tracheal stents. Arch Otolaryngol Head Neck Surg. 2007;133:37-41. This study describes the technical aspects of metallic stent removal, in particular in more complex cases.

6. Food and Drug Administration. FDA public health notification: complications from metallic tracheal stents in patients with benign airway disorders. http://www.fda.gov/MedicalDevices/Safety/ AlertsandNotices/PublicHealthNotifications/ucm062115.htm. Accessed September 15th, 2012.

7. Dineen KM, Jantz MA, Silvestri GS. Tracheobronchial stents. J Bronchol. 2002;9:127-37.

8. Ernst A, Feller-Kopman D, Becker HD, Mehta AC. Central airway obstruction. Am J Respir Crit Care Med. 2004;169:1278-97.

9. Colt HG, Harrell JH. Therapeutic rigid bronchoscopy allows level of care changes in patients with acute respiratory failure from central airways obstruction. Chest. 1997;112:202-6.

10. Oviatt PL, Stather DR, Michaud G, et al. Exercise capacity, lung function, and quality of life after interventional bronchoscopy. J Thorac Oncol. 2011;6:38-42.

11. Herth FJF, Eberhardt R, Becker HD. Effects of self-expanding metal stents on work of breathing in patients with tracheobronchial stenosis. J Bronchol. 2004;11:233-6.
12. Bolliger CT, Mathur PN. ERS/ATS statement on interventional pulmonology. Eur Respir J. 2002;19:356-73.

13. Kvale PA, Selecky PA, Prakash UBS. Palliative care in lung cancer ACCP evidence-based clinical practice guidelines (2nd edition). Chest. 2007;132:368S-403.

14. Chhajed PN, Baty F, Pless M, et al. Outcome of treated advanced nonsmall cell lung cancer with and without central airway obstruction. Chest. 2006;130:1803-7.

15. - Alazemi S, Lunn W, Majid A, et al. Outcomes, health-care resources use, and costs of endoscopic removal of metallic airway stents. Chest. 2010;138:350-6. This study describes one of the single largest experiences with metallic stent removal, and the impact of stent removal on health care utilization.

16. Madden BP, Loke T-K, Sheth AC. Do expandable metallic airway stents have a role in the management of patients with benign tracheobronchial disease? Ann Thorac Surg. 2006;82:274-8.

17. Pastis NJ, Nietert PJ, Silvestri GA. Variation in training for interventional pulmonary procedures among US pulmonary/critical care fellowships a survey of fellowship directors. Chest. 2005;127:1614-162.

18. Ernst A. Metal stenting as first-line therapy for tracheal compression after gastric pull-up. Chest. 2002;122:2269.

19. - Lund ME, Force S. Airway stenting for patients with benign airway disease and the food and drug administration advisory. A call for restraint. Chest. 2007;132:1107-8. This article was written in response to the FDA advisory against the routine use of metallic stents in benign disease. It outlines the particular concerns and recommends against their use unless all other therapeutic options have been exhausted.

20. Rodriguez AN, Diaz-Jimenez JP, Edell ES. Silicone stents versus metal stents for management of benign tracheobronchial disease con: metal stents. J Bronchol. 2000;7:184-7.

21. Saad CP, Murthy S, Krizmanich G, et al. Self-expandable metallic airway stents and flexible bronchoscopy long-term outcomes analysis. Chest. 2003;124:1993-9.

22. Herth FJF, Peter S, Baty F, et al. Combined airway and oesophageal stenting in malignant airway-oesophageal fistulas: a prospective study. Eur Respir J. 2010;36:1370-4.

23. Freitag L, Tekolf E, Steveling H, et al. Management of malignant esophagotracheal fistulas with airway stenting and double stenting. Chest. 1996;110:1155-60.

24. Becker HD. Stenting of the central airways. J Bronchol. 1995;2:98 106.

25. Miyazawa T, Yamakido M, Ikeda S, et al. Implantation of ultraflex nitinol stents in malignant tracheobronchial stenoses. Chest. 2000;118:959-65.

26. Freitag L, Eicker K, Donovan TJ, Dimove D. Mechanical properties of airway stents. J Bronchol. 1995;2:270-8.

27. Shiraishi T, Shirakusa T, Ninomiya H, et al. Penetration of the aortic wall by a metallic airway stent. A successfully treated case with left pneumonectomy and aortic repair. J Cardiovasc Surg (Torino). 2005;46:473-5.

28. Monnier P, Mudry A, Stanzel F, et al. The use of the covered Wallstent for the palliative treatment of inoperable tracheobronchial cancers a prospective, muiticenter study. Chest. 1996;110:1161-8.

29. Lunn W, Feller-Kopman D, Wahidi M, et al. Endoscopic removal of metallic airway stents. Chest. 2005;127:2106-12.

30. Leroy S, Marquette C-H, Freitag L. A new self-expanding, removable, covered metal tracheobronchial stent: results of an animal trial. J Bronchol. 2006;13:111-3.

31. Gildea TR, Downie G, Eapen G, et al. A prospective multicenter trial of a self-expanding hybrid stent in malignant airway obstruction. J Bronchol. 2008;15:221-4.

32. Alazemi S, Chatterji S, Ernst A. Mediastinal migration of selfexpanding bronchial stents in the management of malignant bronchoesophageal fistula. Chest. 2009;135:1353-5. 
33. Swanson KL, Edell ES, Prakash UBS, et al. Complications of metal stent therapy in benign airway obstruction. J Bronchol. 2007;14:90-4.

34. Gottlieb J, Fuehner T, Dierich M, et al. Are metallic stents really safe? A long-term analysis in lung transplant recipients. Eur Respir J. 2009;34:1417-22.

35. Chung F-T, Chen H-C, Chou C-L, et al. An outcome analysis of self-expandable metallic stents in central airway obstruction: a cohort study. J Cardiothorac Surg. 2011;6:46-53.

36. Ost DE, Shah AM, Lei X, et al. Respiratory infections increase the risk of granulation tissue formation following airway stenting in patients with malignant airway obstruction. Chest. 2012;141:1473-81.
37. Nobukiyo S, Iwatake H, Watanabe $\mathrm{S}$, et al. A case of relapsing polychondritis involving placement of an expandable metallic stent. Auris Nasus Larynx. 2003;30(Suppl):S141-4.

38. Urschel JD. Delayed massive hemoptysis after expandable bronchial stent placement. J Laparoendosc Adv Surg Tech A. 1999;9(2):155-8.

39. Noppen M, Stratakos G, D'Haese J, et al. Removal of covered selfexpandable metallic airway stents in benign disorders indications, technique, and outcomes. Chest. 2005;127:482-7.

40. Fruchter O, Raviv Y, Fox BD, et al. Removal of metallic tracheobronchial stents in lung transplantation with flexible bronchoscopy. J Cardiothorac Surg. 2010;5:72-5.

41. Wang H, Zhou Y, Yamaguchi E, et al. Endoscopic removal of metallic airway stents. J Bronchol Intervent Pulmonol. 2011;18:31-7. 